\title{
SYNTHESIS OF SOME NEW BIOACTIVE 1-N-ACID HYDRAZIDE SUBSTITUTED PYRAZOLINES
}

\author{
Sadaf Sadiq Khan and Aurangzeb Hasan* \\ Department of Chemistry, Quaid-i-Azam University, Islamabad 45320, Pakistan \\ flavonoids@hotmail.com
}

\begin{abstract}
A series of 14 new bioactive $1-\mathrm{N}$-acid hydrazide substituted pyrazolines were synthesized by cyclization of variably substituted chalcones and different acid hydrazides, using acetic acid as a solvent. The chemical structure of the compounds was characterized by FTIR, EIMS and ${ }^{~}$ H NMR spectroscopy. The antibacterial activities of these compounds were evaluated by agar well diffusion method. 1-Npicolinic acid hydrazide pyrazoline was found to be more active as compared to the standard antibiotic Roxithromycin.
\end{abstract}

\section{Introduction}

Pyrazolines and their derivatives display various biological properties such as pesticidal, fungicidal, insecticidal, anti-inflammatory, antiarthritic, antidepressant and antiviral activities ${ }^{[1-3]}$. Considerable interest has been focused on the pyrazolines structure, which has known to possess a broad spectrum of biological activities such as antinociceptive, antibacterial, antioxidant and antiamoebic ${ }^{[4-8]}$. Moreover, these heterocyclic compounds, in addition to biological activities have also shown some industrial applications like bleaching agents, dyes, optical brighteners and various fluorescent whitening agents ${ }^{[1,3,9-}$ ${ }^{12]}$. Earlier studies by Gabriele Murineddu, et al. ${ }^{[13]}$ also demonstrated the analgesic and antiinflammatory activities of acid hydrazides.

The present study is therefore devoted to the synthesis of pyrazolines from variably substituted chalcones which are also associated with diverse biological activities ${ }^{[14-18]}$ and different acid hydrazides. This study was carried out in the quest to prepare pyrazolines not synthesized earlier, that may possess new and/or enhanced biological and other industrial properties. We report the synthesis of a series of 14 new 1-Nacid hydrazide substituted pyrazolines which were characterized by physical and FTIR, EIMS and 1H NMR spectroscopic data and have also been evaluated for their antibacterial activities

\section{Results and Discussions}

1,3-Diphenyl-2-propen-1-ones (chalcones) 1a-f were synthesized by condensing acetophenone (i) with appropriate benzaldehyde derivatives (ii) in dilute ethanolic sodium hydroxide solution at room temperature.1-N-acid hydrazide substituted -2-pyrazolines $(2 \mathrm{~g}-\mathrm{t})$ were synthesized by the reaction of appropriate 1,3-Diphenyl-2-propen-1-one derivatives (1a-f) and acid hydrazides (iii) according to the following Scheme-1. Physical data of synthesized pyrazolines (2g-t) are listed in Table-1.
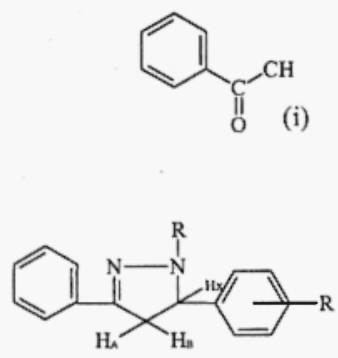

2-pyrazolines (2g-t)

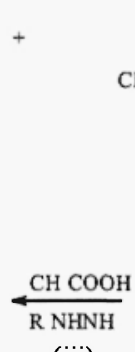

(iii)

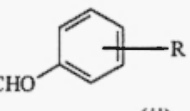

\section{(ii)}<smiles>[R]c1ccc(/C=C/C(=O)c2ccccc2)cc1</smiles>

Chalcones (1a-f)

Scheme-1 
Table-1 : Structure and physical data of pyrazolines (2g-t)

\begin{tabular}{|c|c|c|c|c|c|c|c|}
\hline $\begin{array}{c}\text { Compound } \\
\#\end{array}$ & $\overline{\mathbf{R}_{1}}$ & $\mathbf{R}_{\mathbf{2}}$ & Formula & M.P. ${ }^{0} \mathrm{C}$ & $\begin{array}{l}\text { Yield } \\
(\%)\end{array}$ & $\overline{R_{f}}$ & $\begin{array}{c}\text { Purification } \\
\text { Solvent }\end{array}$ \\
\hline $2 \mathrm{~g}$ & $6-1=0$ & $-\mathrm{H}$ & $\mathrm{C}_{21} \mathrm{H}_{17} \mathrm{~N}_{3} \mathrm{O}$ & $158-160$ & 71 & 89 & Ethanol \\
\hline $2 \mathrm{~h}$ & $5 !$ & $-\mathrm{H}$ & $\mathrm{C}_{21} \mathrm{H}_{17} \mathrm{~N}_{3} \mathrm{O}$ & $146-148$ & 75 & 97 & " \\
\hline $2 \mathrm{i}$ & $0-\infty$ & $-\mathrm{H}$ & $\mathrm{C}_{21} \mathrm{H}_{17} \mathrm{~N}_{3} \mathrm{O}$ & $148-150$ & 76 & 93 & " \\
\hline $2 \mathrm{j}$ & $"$ & $-\mathrm{OCH}_{3}$ & $\mathrm{C}_{22} \mathrm{H}_{19} \mathrm{~N}_{3} \mathrm{O}_{2}$ & $130-132$ & 78 & 87 & $"$ \\
\hline $2 \mathrm{k}$ & $"$ & $-\mathrm{NO} 2$ & $\mathrm{C}_{21} \mathrm{H}_{16} \mathrm{~N}_{4} \mathrm{O}_{3}$ & $136-138$ & 72 & 94 & $"$ \\
\hline 21 & $"$ & $-\mathrm{N}\left(\mathrm{CH}_{3}\right)_{2}$ & $\mathrm{C}_{23} \mathrm{H}_{22} \mathrm{~N}_{4} \mathrm{O}$ & $127-129$ & 72 & 94 & $"$ \\
\hline $2 m$ & $"$ & $-F$ & $\mathrm{C}_{21} \mathrm{H}_{16} \mathrm{~N}_{3} \mathrm{OF}$ & $168-169$ & 88 & 91 & $"$ \\
\hline $2 n$ & $"$ & $-\mathrm{CI}$ & $\mathrm{C}_{21} \mathrm{H}_{16} \mathrm{~N}_{3} \mathrm{OCl}$ & $138-140$ & 87 & 92 & $"$ \\
\hline 20 & 0,0 & $-\mathrm{H}$ & $\mathrm{C}_{29} \mathrm{H}_{24} \mathrm{~N}_{2} \mathrm{O}$ & $170-172$ & 80 & 85 & $"$ \\
\hline $2 p$ & $"$ & $-\mathrm{NO}_{2}$ & $\mathrm{C}_{29} \mathrm{H}_{23} \mathrm{~N}_{3} \mathrm{O}_{3}$ & $120-122$ & 65 & 93 & $"$ \\
\hline $2 q$ & $"$ & $-\mathrm{CI}$ & $\mathrm{C}_{29} \mathrm{H}_{23} \mathrm{~N}_{2} \mathrm{OCl}$ & $98-100$ & 71 & 92 & $"$ \\
\hline $2 r$ & $0^{-\infty}$ & $-\mathrm{H}$ & $\mathrm{C}_{22} \mathrm{H}_{18} \mathrm{~N}_{2} \mathrm{O}$ & $158-160$ & 84 & 91 & $"$ \\
\hline $2 s$ & $"$ & $-\mathrm{NO}_{2}$ & $\mathrm{C}_{22} \mathrm{H}_{17} \mathrm{~N}_{3} \mathrm{O}_{3}$ & 136-138 & 75 & 86 & $"$ \\
\hline $2 t$ & $5 !$ & $-\mathrm{H}$ & $\mathrm{C}_{22} \mathrm{H}_{17} \mathrm{~N}_{2} \mathrm{OF}$ & $128-130$ & 73 & 92 & $"$ \\
\hline
\end{tabular}

1-N-Picolinoyl-3, 5-diphenyl-2-pyrazoline $(\mathbf{2 g})$ was obtained as yellow granules. The molecular formula and molecular weight of the compound $(2 \mathrm{~g})$ are $\mathrm{C}_{21} \mathrm{H}_{17} \mathrm{~N}_{3} \mathrm{O}$ and 327 respectively. The FTIR spectrum showed stretching frequencies at $1583 \mathrm{~cm}^{-1} 1281 \mathrm{~cm}^{-1} 1506 \mathrm{~cm}^{-1} 3034 \mathrm{~cm}^{-1} 1690 \mathrm{~cm}^{-1}$ which are characteristics of $\mathrm{C}=\mathrm{N}, \mathrm{C}-\mathrm{C}, \mathrm{C}=\mathrm{C} \mathrm{Ar}$., $\mathrm{CH}_{2}, \mathrm{C}=\mathrm{O}$ of the aromatic rings respectively.

The mass spectrum of 1-N-Picolinoyl-3, 5-diphenyl-2-pyrazoline (2g) showed characteristics peaks of molecular ion peak $\mathrm{M}$ at $\mathrm{m} / \mathrm{z} 327$ which is found in good agreement with molecular weight. The characteristic peaks at $\mathrm{m} / \mathrm{z} 106$ and 78 appeared due to the formation of the picolinoyl and pyridine segments. The base peak in the mass spectrum appeared at $\mathrm{m} / \mathrm{z} 327$ which is also the molecular ion peak.

The ${ }^{1} \mathrm{H}$ NMR spectrum of 1-N-Picolinoyl-3, 5-diphenyl-2-pyrazoline (2g) showed three doublet of doublets at $3.17 \mathrm{ppm}, 3.77 \mathrm{ppm}, 5.60 \mathrm{ppm}$, with large coupling constant $4.70,17.6 \mathrm{~Hz}, 11.69,17.6 \mathrm{~Hz}$ and $4.71,11.6 \mathrm{~Hz}$ which are characteristic and showed following type of arrangement of protons.<smiles>CC(C)(C)[14CH3]</smiles>

The aromatic protons appeared as a multiplet in the range of 7.23-7.47 ppm. The characteristic peak for the picolinoyl protons appear as doublet of doublet of 4-H between 7.81-10.37. Interpretation of FTIR, mass, NMR, spectral data and physical constants confirmed the structure of ( $2 \mathrm{~g})$ as 1-N-Picolinoyl-3, 5diphenyl-2-pyrazoline. Similarly all other pyrazolines $(\mathbf{2 h - t )}$ were characterized on the basis of their physical and spectral data. 


\section{Experimental Protocols}

All melting points were determined in open capillaries using Gallenkemp melting point apparatus and are un-corrected. All chemicals were supplied by Fluka and Sigma Aldarich (Germany). $\mathrm{R}_{\mathrm{f}}$ values were calculated by using pre-coated silica gel aluminum backed plates Kieselgel $60 \mathrm{~F}_{254}$ Merck (Germany), in ethyl acetate: pet-ether (1:9). FTIR spectra were recorded on Bio-Rad Merlin using $\mathrm{KBr}$ discs. ${ }^{1} \mathrm{H}$ NMR spectra were recorded on GEQE 3400 Spectrometer (Oxford Magnet) using TMS as internal standard. EIMS were recorded on VG: 70 SE Mass Spectrometer. Purity of each compound was monitored by TLC.

\section{General Procedure for the Preparation of 1, 3-Diphenyl-2-propen-1-ones (1a)}

1, 3-Diphenyl-2-propen-1-one derivatives were synthesized by condensing acetophenone $(0.01 \mathrm{~mol})$ with appropriate benzaldehyde derivatives $(0.01 \mathrm{~mol})$ according to Claisen Schmidt condensation reaction ${ }^{[19-20]}$

\section{General Procedure for the Preparation of 1-N-picolinoyl-1, 3-Diphenyl-2-pyrazoline (2g)}

Picolinic acid hydrazide $(0.013 \mathrm{~mol}) 1.78 \mathrm{~g}$ was added to 1,3-Diphenyl-2-propen- 1-one (chalcone) 1a-f $(0.01 \mathrm{~mol}) 2.08 \mathrm{~g}$ in acetic acid $(15 \mathrm{ml})$ and the mixture was refluxed under constant stirring for 3 hours at $100-110^{\circ} \mathrm{C}$ until the cyclization is completed and a deep orange colour developed. The reaction mixture was diluted with ice-cold water, extracted with ethyl acetate and the solvent was removed under reduced pressure. This procedure afforded the product $2 \mathrm{~g}$ which was purified by crystallization from ethanol. IR $(\mathrm{KBr})\left(\mathrm{v}_{\max } / \mathrm{cm}^{\mathrm{l}}\right): 1585,1281,1506,3034,1690 .{ }^{1} \mathrm{H} \mathrm{NMR}\left(\mathrm{CDCl}_{3}\right) \delta \mathrm{H}: 3.17(\mathrm{dd}, 1 \mathrm{H}, \mathrm{HA}), 3.77(\mathrm{dd}, 1 \mathrm{H}$, $\left.\mathrm{H}_{\mathrm{B}}\right)$, 5.60(dd, $\left.1 \mathrm{H}, \mathrm{H}_{\mathrm{X}}\right), 7.237 .47\left(\mathrm{ml0H}, \mathrm{H}_{\text {arom. }}\right)$, 7.81-10.37(dds, $\left.4 \mathrm{H}, \mathrm{H}_{\text {picolinoyl }}\right)$. EIMS (m/z, \%): 327( $\mathrm{M}^{+}$, 11.9), 221(28.2), 106(12.2), 78(100), 51(15.3).

1-N-Isonicotinoyl-1, 3-Diphenyl-2-pyrazoline (2h) IR (KBr) $\left(v_{\max } / \mathrm{cm}^{1}\right): 1593,1302,1491,3000,1636$. ${ }^{1} \mathrm{HNMR}\left(\mathrm{CDCl}_{3}\right) \delta \mathrm{H}: 3.27\left(\mathrm{dd}, 1 \mathrm{H}, \mathrm{H}_{\mathrm{A}}\right), 3.84\left(\mathrm{dd}, 1 \mathrm{H}, \mathrm{H}_{\mathrm{B}}\right), 5.80\left(\mathrm{dd}, 1 \mathrm{H}, \mathrm{H}_{\mathrm{X}}\right), 7.23-7.43\left(\mathrm{~m} \mathrm{lOH}_{1} \mathrm{H}_{\text {arom }}\right.$ ), 7.6-8.76 (dds, $4 \mathrm{H}, \mathrm{H}_{\text {isoncotinoyl }}$ ). EIMS (m/z, \%): 327(M+100), 221(21.2), 106(68), 78(32.4), 51(16).

1-N-Nicotinoyl-1, 3-Diphenyl-2-pyrazoline (2i) IR (KBr) $\left(v_{\max } / \mathrm{cm}^{1}\right): 1587,1200,1476,3197,1346$. ${ }^{1} \mathrm{HNMR}\left(\mathrm{CDCl}_{3}\right) \delta \mathrm{H}: 3.27(\mathrm{dd}, 1 \mathrm{H}, \mathrm{HA}), 3.84\left(\mathrm{dd}, 1 \mathrm{H}, \mathrm{H}_{\mathrm{B}}\right), 5.82(\mathrm{dd}, 1 \mathrm{H}, \mathrm{HX}), 7.23-7.42\left(\mathrm{dd}, 1 \mathrm{H}, \mathrm{H}_{\mathrm{X}}\right), 7.23-$ 7.42(10H, m, $\left.\mathrm{H}_{\text {arom }}\right), 7.71-8.68,9.29\left(2 \mathrm{H}, 2 \mathrm{ds}, 1 \mathrm{H}, \mathrm{s}, \mathrm{H}_{\text {nicotinoyl }}\right)$. EIMS (m/z, \%): 327( $\left.\mathrm{M}^{+}, 11.9\right), 106(100)$, 78(43.2), 51(10.8).

1-N-Nicotinoyl-3-phenyl-5-(p-methoxyphenyl)-2-pyrazoline $(2 \mathrm{j}) \operatorname{IR}(\mathrm{KBr})\left(\mathrm{v}_{\max } / \mathrm{cm}^{1}\right): 1595,1230$, $1495,3090,1650 .{ }^{1} \mathrm{HNMR}\left(\mathrm{CDCl}_{3}\right) \delta \mathrm{H}: 3.26\left(\mathrm{dd}, 1 \mathrm{H}, \mathrm{H}_{\mathrm{A}}\right), 3.80\left(\mathrm{dd}, 1 \mathrm{H}, \mathrm{H}_{\mathrm{B}}\right), 5.80\left(\mathrm{dd}, 1 \mathrm{H}, \mathrm{H}_{\mathrm{X}}\right), 7.23-$ $7.40\left(9 \mathrm{H}, \mathrm{m}, \mathrm{H}_{\text {arom }}\right), 7.70-8.68,9.20\left(2 \mathrm{H}, 2 \mathrm{ds}, 1 \mathrm{H}, \mathrm{s}, \mathrm{H}_{\text {nicotinoyl }}\right), 3.70\left(3 \mathrm{H}, \mathrm{s}, \mathrm{OCH}_{3}\right)$. EIMS $(\mathrm{m} / \mathrm{z}, \%): 357($ $\left.\mathrm{M}^{+}, 40\right), 106(100), 78(47), 51(12)$.

1-N-Nicotinyl-3-phenyl-5-(m-nitrophenyl)-2-pyrazoline (2k) IR (KBr) $\left(v_{\max } / \mathrm{cm}^{1}\right): 1580,1190,1480$, 3102, and 1630. ${ }^{1} \mathrm{HNMR}\left(\mathrm{CDCl}_{3}\right) \delta \mathrm{H}: 3.26\left(\mathrm{dd}, 1 \mathrm{H}, \mathrm{H}_{\mathrm{A}}\right), 3.81\left(\mathrm{dd}, 1 \mathrm{H}, \mathrm{H}_{\mathrm{B}}\right), 5.80\left(\mathrm{dd}, 1 \mathrm{H}, \mathrm{H}_{\mathrm{X}}\right), 7.23-$ 7.41 $\left(9 \mathrm{H}, \mathrm{m}, \mathrm{H}_{\text {arom }}\right), 7.72-8.80,9.22\left(2 \mathrm{H}, 2 \mathrm{ds}, 1 \mathrm{H}, \mathrm{s}, \mathrm{H}_{\text {ncotunoyl }}\right)$. EIMS $(\mathrm{m} / \mathrm{z}, \%): 372\left(\mathrm{M}^{+}, 39\right), 106(100)$, $78(50), 51(14)$.

1-N-Nicotinyl-3-phenyl-5-(p-N, N-dimethylphenyl)-2-pyrazoline (2l) $\mathrm{IR}(\mathrm{KBr})\left(\mathrm{v}_{\max } / \mathrm{cm}^{\mathrm{l}}\right): 1597,1225$, $1496,3159,1644$, and $1300 .{ }^{1} \mathrm{HNMR}\left(\mathrm{CDCl}_{3}\right) \delta \mathrm{H}: 3.36\left(\mathrm{dd}, 1 \mathrm{H}, \mathrm{H}_{\mathrm{A}}\right), 3.91\left(\mathrm{dd}, 1 \mathrm{H}, \mathrm{H}_{\mathrm{B}}\right), 5.86\left(\mathrm{dd}, 1 \mathrm{H}, \mathrm{H}_{\mathrm{X}}\right)$, $7.23-7.50\left(9 \mathrm{H}, \mathrm{m}, \mathrm{H}_{\text {arom. }}\right), 7.73-8.78,9.30\left(2 \mathrm{H}, 2 \mathrm{ds}, 1 \mathrm{H}, \mathrm{s}, \mathrm{H}_{\text {nicotinoyl }}\right), 2.91\left(6 \mathrm{H}, \mathrm{s}, \mathrm{N}\left(\mathrm{CH}_{3}\right)_{2}\right)$. EIMS $(\mathrm{m} / \mathrm{z}$, $\%): 370\left(\mathrm{M}^{+}, 50\right), 106(100), 78(47), 51(20)$. 
1-N-Nicotinoyl-3-phenyl-5-(m-fluorophenyl)-2-pyrazoline (2m) IR $(\mathrm{KBr})\left(v_{\max } / \mathrm{cm}^{1}\right): 1575,1187$, $1476,3170,1632$, and $1120 .{ }^{1} \mathrm{HNMR}\left(\mathrm{CDCl}_{3}\right) \delta \mathrm{H}: 3.35\left(\mathrm{dd}, 1 \mathrm{H}, \mathrm{H}_{\mathrm{A}}\right), 3.89\left(\mathrm{dd}, 1 \mathrm{H}, \mathrm{H}_{\mathrm{B}}\right), 5.80\left(\mathrm{dd}, 1 \mathrm{H}, \mathrm{H}_{\mathrm{X}}\right)$, 7.30-7.40(9H, m, $\left.\mathrm{H}_{\text {arom }}\right), 7.70-8.70,9.29\left(2 \mathrm{H}, 2 \mathrm{ds}, 1 \mathrm{H}, \mathrm{s}, \mathrm{H}_{\text {nicotinoyl }}\right)$. EIMS (m/z, \%): 345( $\left.\mathrm{M}^{+}, 48\right)$, 106(100), 78(51), 51(20).

1-N-Nicotinyl-3-phenyl-5-(p-chlorophenyl)-2-pyrazoline $(2 \mathrm{n}) \mathrm{IR}(\mathrm{KBr})\left(v_{\max } / \mathrm{cm}^{1}\right): 1582,1220,1482$, 3140, 1660, 695. ${ }^{1} \mathrm{HNMR}\left(\mathrm{CDCl}_{3}\right) \delta \mathrm{H}: 3.37\left(\mathrm{dd}, 1 \mathrm{H}, \mathrm{H}_{\mathrm{A}}\right), 3.86\left(\mathrm{dd}, 1 \mathrm{H}, \mathrm{H}_{\mathrm{B}}\right), 5.82\left(\mathrm{dd}, 1 \mathrm{H}, \mathrm{H}_{\mathrm{X}}\right), 7.29-$ 7.45(9H, m, $\left.\mathrm{H}_{\text {arom }}\right), 7.69-8.82,9.30\left(2 \mathrm{H}, 2 \mathrm{ds}, 1 \mathrm{H}, \mathrm{s}, \mathrm{H}_{\text {nicotinoyl }}\right)$. EIMS (m/z, \%): 361( $\left.\mathrm{M}^{+}, 60\right), 106(100)$, $78(60), 51(40)$.

1-N-Benzeliloyl-3, 5-diphenyl-2-pyrazoline (2o) IR (KBr) $\left(v_{\max } / \mathrm{cm}^{1}\right):$ 1536, 1298, 1490, 3034, 1697. ${ }^{1} \mathrm{HNMR}\left(\mathrm{CDCl}_{3}\right) \delta \mathrm{H}: 3.25\left(\mathrm{dd}, 1 \mathrm{H}, \mathrm{H}_{\mathrm{A}}\right), 3.78\left(\mathrm{dd}, 1 \mathrm{H}, \mathrm{H}_{\mathrm{B}}\right), 5.80\left(\mathrm{dd}, 1 \mathrm{H}, \mathrm{H}_{\mathrm{X}}\right), 4.30\left(1 \mathrm{H}, \mathrm{s}, \mathrm{H}_{\text {Aliphatic }}\right), 7.23-$

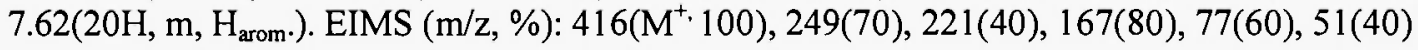

1-N-Benzeliloyl-3-phenyl-5-(m-nitrophenyl)-2-pyrazoline (2p) $\mathrm{IR}(\mathrm{KBr})\left(\mathrm{v}_{\max } / \mathrm{cm}^{1}\right): 1550,1282,1485$, 3020, 1695. ${ }^{1} \mathrm{HNMR}\left(\mathrm{CDCl}_{3}\right) \delta \mathrm{H}: 3.28\left(\mathrm{dd}, 1 \mathrm{H}, \mathrm{H}_{\mathrm{A}}\right), 3.79\left(\mathrm{dd}, 1 \mathrm{H}, \mathrm{H}_{\mathrm{B}}\right), 5.92\left(\mathrm{dd}, 1 \mathrm{H}, \mathrm{H}_{\mathrm{X}}\right), 4.32(1 \mathrm{H}, \mathrm{s}$, $\left.\mathrm{H}_{\text {Aliphatic }}\right), 7.21-7.61\left(20 \mathrm{H}, \mathrm{m}, \mathrm{H}_{\text {arom }}\right)$. EIMS (m/z, \%): 461( $\left.\mathrm{M}^{+}, 100\right), 294(68), 266(42), 167(82), 77(68)$, $51(42)$.

1-N-Benzeliloyl -3-phenyl-5-(4-chlorophenyl)-2-pyrazoline (2q) IR (KBr) $\left(v_{\max } / \mathrm{cm}^{\prime}\right): 1570,1295$, 1499, 3035, 1680, 695. ${ }^{1} \mathrm{HNMR}\left(\mathrm{CDCl}_{3}\right) \delta \mathrm{H}: 3.26\left(\mathrm{dd}, 1 \mathrm{H}, \mathrm{H}_{\mathrm{A}}\right), 3.82\left(\mathrm{dd}, 1 \mathrm{H}, \mathrm{H}_{\mathrm{B}}\right), 5.84\left(\mathrm{dd}, 1 \mathrm{H}, \mathrm{H}_{\mathrm{X}}\right)$, $4.29\left(1 \mathrm{H}, \mathrm{s}, \mathrm{H}_{\text {Aliphatic }}\right), 7.21-7.65\left(20 \mathrm{H}, \mathrm{m}, \mathrm{H}_{\text {arom! }}\right)$. EIMS (m/z, \%): 450( $\left.\mathrm{M}^{+} \cdot 100\right), 283(60), 255(38), 167(90)$, 77(70), 51(32).

1-N-Benzoyl-3,5-diphenyl-2-pyrazoline (2r) IR (KBr) $\left(v_{\max } / \mathrm{cm}^{1}\right): 1594,1244,1493,3060,1690$. ${ }^{1} \mathrm{HNMR}\left(\mathrm{CDCl}_{3}\right) \delta \mathrm{H}: 3.23\left(\mathrm{dd}, 1 \mathrm{H}, \mathrm{H}_{\mathrm{A}}\right), 3.81\left(\mathrm{dd}, 1 \mathrm{H}, \mathrm{H}_{\mathrm{B}}\right), 5.83(\mathrm{dd}, 1 \mathrm{H}, \mathrm{HX}), 7.27-7.30(10 \mathrm{H}, \mathrm{m}, \mathrm{H}$ arom.),7.37-7.88(5H, m, $\left.\mathrm{H}_{\text {Benzoyl }}\right)$. EIMS (m/z, \%): 326(M $\left.\mathrm{M}^{+}, 100\right), 221(27.2), 105(100), 222(26), 77(59.1)$.

1-N-Benzoyl-3-phenyl-5-(m-nitrophenyl)-2-pyrazoline (2s) IR ( $\mathrm{KBr})\left(\mathrm{v}_{\max } / \mathrm{cm}^{\mathrm{l}}\right): 1590,1250,1497$, 3030, 1695. ${ }^{1} \mathrm{HNMR}\left(\mathrm{CDCl}_{3}\right) \delta \mathrm{H}: 3.25\left(\mathrm{dd}, 1 \mathrm{H}, \mathrm{H}_{\mathrm{A}}\right), 3.89\left(\mathrm{dd}, 1 \mathrm{H}, \mathrm{H}_{\mathrm{B}}\right), 5.85\left(\mathrm{dd}, 1 \mathrm{H}, \mathrm{H}_{\mathrm{X}}\right), 7.27-7.50(9 \mathrm{H}$, $\left.\mathrm{m}, \mathrm{H}_{\text {aron }}\right)$ ), 7.37-7.89(5H, m, $\mathrm{H}_{\text {Benzoyl }}$ ). EIMS (m/z, \%): 371(80), 266(28.2), 105(100), 222(26), 77(54).

1-N-(3-Fluorobenzoyl)-3,5-diphenyl-2-pyrazoline (2t) IR (KBr) $\left(v_{\max } / \mathrm{cm}^{1}\right): 1596,1540,1480,3025$, 1685, 1130. ${ }^{1} \mathrm{HNMR}\left(\mathrm{CDCl}_{3}\right) \delta \mathrm{H}: 3.24\left(\mathrm{dd}, 1 \mathrm{H}, \mathrm{H}_{\mathrm{A}}\right), 3.87\left(\mathrm{dd}, 1 \mathrm{H}, \mathrm{H}_{\mathrm{B}}\right), 5.81\left(\mathrm{dd}, 1 \mathrm{H}, \mathrm{H}_{\mathrm{X}}\right), 7.23-7.50(10 \mathrm{H}$, $\mathrm{m}, \mathrm{H}$ arom.), 7.62-7.84(4H, m, $\mathrm{H}_{\text {Benzoyl }}$ ). EIMS (m/z, \%): 344(70.3), 221(9.7), 123(100), 240(18.3), 95(20.4).

\section{Antibacterial Activity Analysis}

Synthesized compound were tested for their antibacterial activity by adopting agar well diffusion method [21]. Bacteria cultures used were Escherichia coli, Bacillas subtilis, Pseudomonas pickiti, Enterobacter aerogenes and Micrococcus luteus, Roxithromycin was used as standard drug. Using $100 \mu$ micropipette, test solutions were poured in respective wells. Different concentrations of test samples, a solution for positive control (Roxithromycin) and one for negative control (DMSO) was applied to each Petri plate. These plates were incubated at $37^{\circ} \mathrm{C}$. After 24 hours and 48 hours of incubation the diameter of the clear zones, showing no bacterial growth, around each well was measured. Triplicate plates were prepared for each sample compound. Mean clear zone of these plates was calculated in $\mathrm{mm}$ with standard deviation (Table-2). 
Table-2: Antibacterial activity of pyrazolines (2g-t) against five bacterial strains.

\begin{tabular}{|l|c|c|c|c|c|c|}
\hline \multirow{2}{*}{ Compound } & \multicolumn{5}{|c|}{ Zone of Inhibition (mm) } & Remarks/ \\
Activity
\end{tabular}

Pyrazoline $\mathbf{2 g}$ was found to be significantly active against Escherichia coli, Bacillas subtilis and Pseudomonas pickiti bacterial strains. However, the same pyrazoline exhibited even stronger antibacterial activity against Enterobacter aerogenes and Micrococcus luteus as compared to the standard drug Roxithromycin. Most of the other pyrazolines (2h-t) showed some activity only against Pseudomonas pickiti and Enterobacter aerogenes. Strong antibacterial activity of pyrazoline $\mathbf{2} \mathbf{g}$ may be due to the relative position of the $\mathrm{N}$-atom with respect to the carbonyl group of the acid hydrazide.

\section{Conclusions}

It is concluded that among different pyrazolines the presence of different hydrazide moieties, various substituents and their positions are very important from biological activity point of view. Pyrazoline $\mathbf{2 g}$ was found to be significantly active against Escherichia coli, Bacillas subtilis and Pseudomonas pickiti bacterial strains. However, the same pyrazoline exhibited even stronger antibacterial activity against Enterobacter aerogenes and Micrococcus luteus as compared to the standard drug Roxithromycin. Most of the other pyrazolines (2h-t) showed some activity only against Pseudomonas pickiti and Enterobacter aerogenes. Strong antibacterial activity of pyrazoline $\mathbf{2} \mathrm{g}$ may be due to the relative position of the $\mathrm{N}$-atom with respect to the carbonyl group of the acid hydrazide 


\section{References}

1. R.V. Krishnakumar, V. Vijayabasker, S. Perumal, S. Selvaraj and S. Natarajan, Acta Cryst., 60, 879 (2004).

2. Z.Y. Wang, H.J. Shi and H.X. Shi, Hecheng Huaxue, 3, 226 (1995) (In Chinese).

3. M.A. El-Hashash, E.M.A. Soiman, Z.M. Souka and A.S.S. Salman, Rev. Roum. Chim., 40, 59 (1995).

4. Z. Tabarelli, M.A. Rubin, D.B. Berlese, P.D. Sauzem, T.P. Missio, M.V. Teixeira, A.P. Sinhorin, and J. Braz, Med. Biol. Res., 37, 1531 (2004).

5. M.A. Berghot and F.B. Moawad, Eur. J. Pharm. Sci., 20, 173 (2003).

6. T.S. Jeong, K.S. Kim, J.R. Kim, K.H. Cho, S. Lee and W.S. Lee, Biorg. Medic. Chem. Lett., 14, 2719 (2004).

7. N.Soni, K. Pande., R. Kalsi, T.K. Gupta, S.S. Parmer and J.P. Barthwal, Res. Commun. Chem. Pathol. Pharm. 56, 129 (1987).

8. M. Abid and A. Azam, Bioorganic \& Med. Chem., 13, 2213 (2005).

9. T. Eicher and Hauptmann, "The Chemistry of Heterocycles", George Thieme Verlog, Stuttgart, New York (1995).

10. G.M. Badger, "The Chemistry of Heterocycles", Academic Press, New York, London (1961).

11. R.M. Acheson, "An Introduction to the Chemistry of Heterocyclic Compounds., $3^{\text {rd }}$ Ed., John Wiley \& Sons, New York (1976).

12. S.G. Kücükgüzel and S. Rollas, II Farmaco, 47, 583 (2002).

13. G. M.urineddu., G. Loriga., E. Gavin., A. T. Peana, A. C. Mule and G. A. Pinna., Issu 12, 393 (2002).

14. D.A. Jacob, G. Lal, M.M. Sharma, A. Singh, A. Curr Sci., 48, 961 (1979).

15. N.A.Abdou, K.M.Youssef, and M.M.Kandeel, Bull. Fac. Pharm., 31, 361 (1993).

16. A.Syed, H.A.Eman and M.R.Selim, Pak. J. Sci. Ind. Res., 39, 14 (1996).

17. T.Sninomiya, H.Kaminaga, S.Nozaki and M. Sakamoto, Chem. Abstr., 1998, 50388u.

18. J.Michael, A. Masood, J. Alanzi and C. Wright,. J. Heterocyclic Chem., 35, 65 (1998).

19. Kohler H. M., Chadwell H., in: Gilman H., Blatt A. H., (Eds). "Organic Syntheses", Wiley, New York, 1967, p.78 (Coll. Vol. 1).

20. H. S. Mehra, J. Indian Chem. Soc., 45, 178 (1968).

21. M. Leven, B. Vanden, F. Mertens, A. Vlietinck and E. Lammens, Planta Medica, 36, 311 (1979)

Received on February 7, 2006 